\title{
NUMERICAL ANALYSIS OF NON-LINEAR LARGE-STRAIN CONSOLIDATION AND FILLING
}

\author{
A. Huerta and A. RODRiguez \\ Departamento de Matemática Aplicada III, Escuela Técnica Superior de Ingenieros de Caminos, \\ Canales y Puertos, Universitat Politècnica de Catalunya, Jordi Girona Salgado 31, \\ 08034 Barcelona, Spain
}

\begin{abstract}
Finite strain consolidation and filling of soft sediments at high water level is a challenging problem because of its highly non-linear physical and mathematical aspects. Several numerical schemes designed for this problem are presented as well as simple numerical improvements for a better handling of the extremely high variations of the material properties with depth. The numerical algorithms developed are robust and verify convergence of the iterative schemes instead of the more classical approaches based or choosing time increments 'sufficiently' small and assuming convergence at every step.

A set of computer programs has been developed to predict magnitude and rate of large-strain self-weight one-dimensional and pseudo bi-dimensional (i.e. one-dimensional deformation, bi-dimensional flux) consolidation during and after deposition, that is, coupling filling and consolidation phenomena. The actual life of the deposit can be numerically simulated combining filling periods and quiescent periods where surcharges (or capping) can exist. Consequently, they are a basic technique for the design of disposal ponds.
\end{abstract}

\section{INTRODUCTION}

Whe prediction of densification magnitudes and rates 1.t. major concern in the disposal of fine grained aturned mineral wastes, such as mine tailings, Wumina red mud, oil sand sludge, dredged materials, etc. The primary issues center around predicting the time-dependent capacity of a given disposal area-as Well as ultimate storage capacity and useful life-and The time-rate improvement of material properties for reciamation purposes. Intrinsic to both issues is the process of consolidation and the associated material extinges.

(a)

A set of computer programs has been developed 1. predict magnitude and rate of large-strain selfWeight consolidation during and after deposition, that is; coupling filling and consolidation phenometia. The actual life of the deposit can be numerically simulated combining filling periods and quiescent periods.

Due to the loose nature of the sedimented deposit (solid contents ranging from less than 10\% up to $30 \%$ ), the ensuing vertical settlements are usially extremely large and beyond the range that can bee handled by classical small-strain consolidation theories; accordingly non-linear finite deformation ntoodels must be developed. Since the physical and mathematical aspects of this problem are highly non-linear, the numerical algorithms developed verify convergence of the iterative schemes instead of the more classical approaches based on choosing ofime increments 'sufficiently' small and assuming convergence at every step. This procedure precludes situmerical aberrations that appear with other abproaches and increases accuracy in the first half of the consolidation process. It should be noted that due to the alternation between periods of filling and quiescent consolidation good accuracy at the first or middle stages of consolidation is needed, because the results obtained after a cycle are the initial conditions for the following one. This is not the case of classical consolidation analyses concerned mostly with the final stages of consolidation.

The next section contains a brief background of finite-strain theory and a discussion of the material relationships employed. This is followed by a statement of the numerical problem together with the pertinent algorithms studied and developed. Next a couple of one-dimensional examples and the bi-dimensional extension of the problem are presented.

\section{THEORETICAL CONSIDERATIONS}

The theory presented here is patterned after the early work by Gibson et al. [1], and several modifications that are described in [2-7] among others. Two standard formulations for the consolidation equation are employed in finite-strain theory, depending upon whether the void ratio, $e$, see for instance $[1,2,4,5,8]$, or the excess pore-water pressure, $u$, see $[3,9]$, is selected as the dependent variable. The approach adopted here uses excess over hydrostatic pore-water pressure, $u$, as the dependent variable. The one-dimensional consolidation equation obtained is [3]:

$$
\frac{\partial}{\partial z}\left[-\frac{k}{\gamma_{w}(1+e)} \frac{\partial u}{\partial z}\right]+\frac{\mathrm{d} e}{\mathrm{~d} \sigma^{\prime}}\left[\frac{\partial \sigma_{b}}{\partial t}-\frac{\partial u}{\partial t}\right]=0
$$


in which the material coordinate $z$ is given by

$$
z(a)=\int_{0}^{a} \frac{\mathrm{d} a^{\prime}}{1+e_{0}\left(a^{\prime}\right)}
$$

where $a$ is the initial vertical (Lagrangian) coordinate, $e_{0}$ is the initial void ratio, $k$ is the coefficient of permeability, $\gamma_{w^{\prime}}$ is the unit weight of water, $t$ is time, and $\sigma^{\prime}$ and $\sigma_{b}$ are the effective stress and buoyant stress; respectively. The material coordinate, $z$, can be interpreted as the height of solid particles between the planes determined by $a=0$ and $a$, which is constant with time.

The buoyant stress is defined as the total stress minus the hydrostatic pressure, that is

$$
\sigma_{b}(z, t)=\left(\gamma_{s}-\gamma_{w^{\prime}}\right)\left(z_{\text {top }}(t)-z\right)+q_{\text {sur }},
$$

where $\gamma_{s}$ is the unit weight of the solids, $q_{\text {sur }}$ is the surcharge or capping load, and $z_{\text {top }}$ is the material coordinate corresponding to the ton laver of the deposit. Since the surcharge, $q_{\text {sur }}$ is usually assumed to be deposited instantaneously, the buoyant stress depends only on time through $z_{100}$. That is, the variations of the buoyant stress are directly dependent on the increase of the total height of solid particles, i.e. any additional deposition of solid particles, namely the filling rate.

\section{tem $\partial \sigma_{b} / \partial t$, in eqn $(1)$, which of filling and quiescent consol The resolution of eqn fication of boundary and} boundary condition
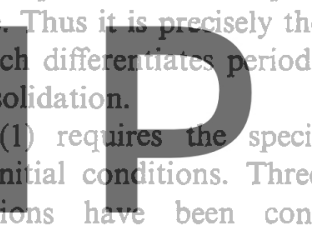

sensititivity analysis conducted with the developed programs concluded that the influence of $\sigma_{0}^{\prime}$ in settlement is negligible and stays under $5 \%$ for the material property distributions, as advanced in [11], Physically, this should be expected since the initial void ratio which arbitrarily represents the limit between sedimentation and consolidation is usually very large [10] and this implies a small $\sigma_{0}^{\prime}$. Other definitions, such as,

$$
\begin{array}{ll}
\sigma^{\prime}=\sigma_{0}^{\prime} & \text { if } \sigma_{b}-u \leqslant \sigma_{0}^{\prime} \\
\sigma^{\prime}=\sigma_{b}-u & \text { if } \sigma_{b}-u>\sigma_{0}^{\prime}
\end{array}
$$

induce unrealistic situations in the upper part of the deposit; namely, constant distributions of void ratio and permeability, thus no consolidation.

It should be noted that sand mixing is easily introduced by assuming that the clay phase governs. both the mechanical and flux behavior of the mixture; That is in the material relationshins a clay void ratio (volume of voids divided by volume of clay) is used instead of the classical void ratio (volume of voids divided by volume of solids).

Finally, it should be noted that the coefficients $k /(1+e)$ and $\mathrm{de} / \mathrm{d} \sigma^{\prime}$ in eqn (1) depend ultimately on the excess pore-water pressure, $u$. Both permeability $E D / A$

sidered: drained, undrained or prescribed piezometric

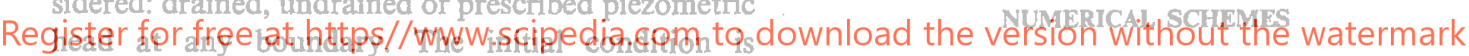

the distribution of excess pore-water pressure with depth.

Appropriate relationships between void ratio and effective stress and between the coefficient of permeability and the void ratio must be implemented. The computer programs developed include several specific types of equations proposed for these two relationships, see $[6,10]$. Available test data on fine grained materials at low solid contents suggest highly non-linear $e-\sigma^{\prime}$ and $k-e$ behaviors which are usually written as power laws.

One important drawback of the usual compressibility relations is that zero effective stress implies infinite void ratio. To avoid this problem without changing the compressibility relationship and the fact that at the drained surface the buoyant stress and the excess pore-water pressure are zero, the effective stress is defined as:

$$
\sigma^{\prime}=\sigma_{b}-u+\sigma_{0}^{\prime}
$$

where $\sigma_{0}^{\prime}$ is obtained by introducing in the compressibility relation the initial void ratio, $e_{0}$. The validity of this assumption is discussed in [11] for steadystate seepage-induced consolidation; moreover, a
A fully implict finite difference method was chosen for the solution of eqn (1) because of its stability: However, most of the conclusions observed are inde pendent of the particular method employed and cañ be generalized to other techniques such as the finite element method.

The dimensionless form of eqn (1) is given by

$$
p\left[\frac{\partial u}{\partial t}-\frac{\partial \sigma_{b}}{\partial t}\right]=\frac{\partial}{\partial z}\left[q \frac{\partial u}{\partial z}\right],
$$

where the same symbols are used for dimensionless variables in order to simplify the expressions. The coefficients $p$ and $q$ in eqn (6) can be interpreted 25 the dimensionless compressibility and permeability: therefore, they are functions of $u$.

After discretization, the resulting system of equat tions is non-linear and given by

$$
\mathbf{A}\left(\mathbf{u}^{n+1}\right) \mathbf{u}^{n+1}=\mathbf{u}^{n}+\mathbf{f},
$$

where $\mathbf{a}^{n+1}$ and $\mathbf{u}^{n}$ are the unknown nodal vectors of excess pore-water pressure at time $t^{n+1}$ and $t^{n}$, respectively, $\mathbf{A}$ is the tridiagonal matrix whose coefficients are function of $p$ and $q$ (this function 


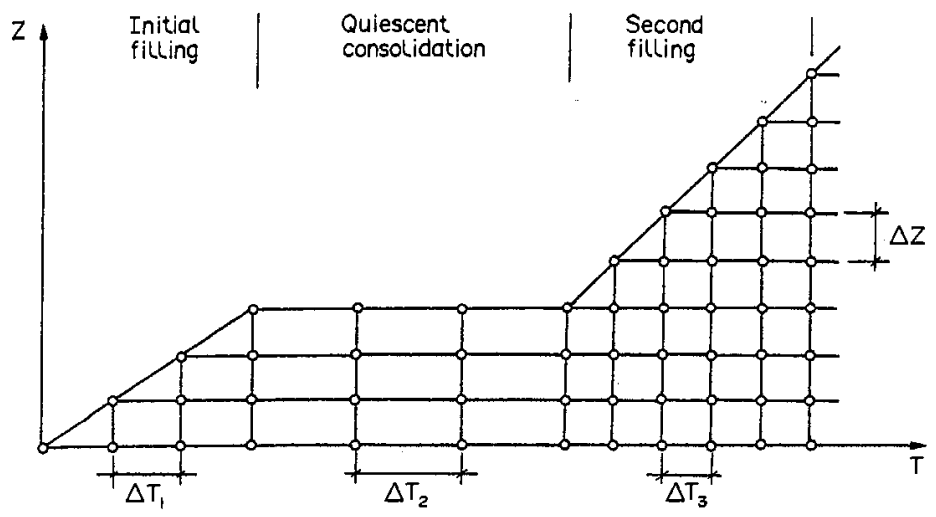

Fig. 1. Domain discretization (one node added per time-step).

depends on the discretization scheme implemented), and $f$ is the nodal vector of increments in the buoyant stress for filling periods ( $f=0$ in the quiescent consolidation periods).

First, the domain discretization is discussed because of the difficulties encountered during the filling periods particularly in the upper areas of the deposit. Then, several approaches for solving eqn (7) are presented. Due to the highly non-linear behavior of the problem, finding the solution of eqn (7) is not an easy task.

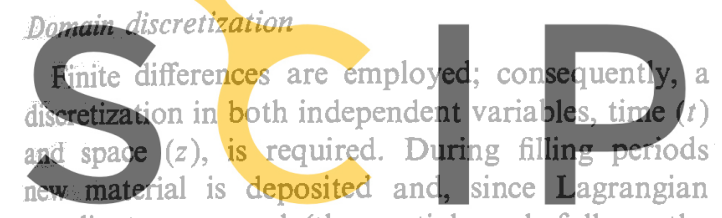
coordinates are used (the spatial mesh follows the

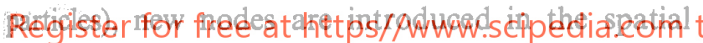
discretization.

Figure 1 shows a classical [3] domain discretization where one node is added per time-step during filling periods. This is a usual and simple technique where the filling rate is the spatial increment divided by the time-step (recall that $z$ is the height of solid particles). However, the strong dependency between both increments is sometimes a serious drawback.

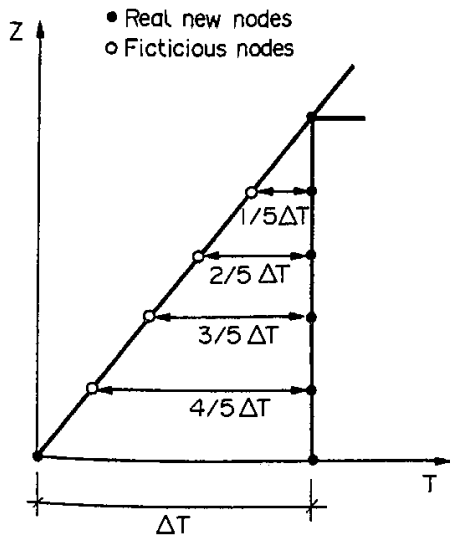

Fig. 2. Multiple node technique per 'deposited' layer.
Self-weight consolidation probltuss present extremely high variations of permeability and compressibility with depth in the upper area of the deposit. This requires a very fine spatial mesh able to capture the changes in material properties; recall that linear interpolation (two nodes per layer) is employed in every 'deposited' layer. The ensuing time-steps are usually very small and the computer cost clearly uneconomical.

If several nodes are introduced per 'deposited' layer (time-step), as shown in Fig. 2, the high variations of compressibility and permeability are better captured and both disoxetizations ( $t$ and $z$ ) are inde-
pendent of each other. To illustrate it, Fig. 3 shows
the void ratio distributions after the first time-step
of an initial filling period over an undrained bed.
As expected, the five nedes per time increment conpares much better than the one node technique with

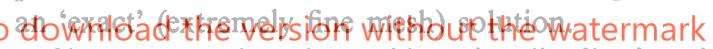

The area under the void ratio distribution is directly related to the depth of the deposit. The over-estimation of $e$ with the one node per time-step discretization induces an under-estimation of settlements, while the multiple node technique developed compensates the areas and consequently predicts settlements much more accurately. This is an

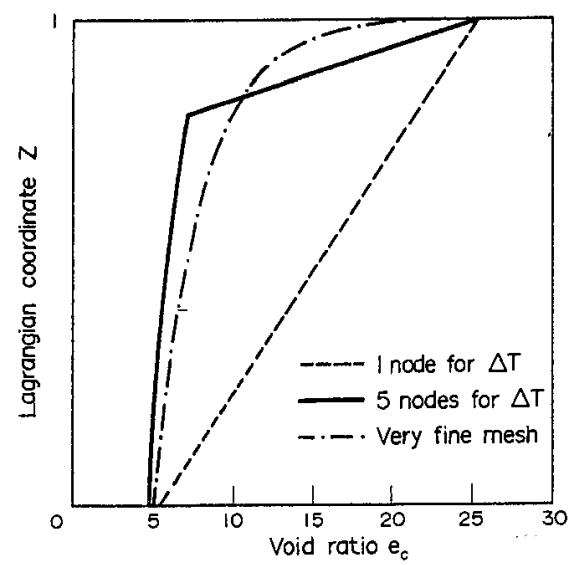

Fig. 3. Comparison between one node and multiple node techniques. 
important feature that will be clearly observed in the presented examples.

\section{Linearization of the problem}

In order to solve the non-linear set of algebraic eqns (7), the classical approaches [3] linearize the problem replacing in the matrix $\mathbf{A}$ the solution of the last time increment, i.e. they assume $\mathbf{A}\left(\mathbf{u}^{n+1}\right)=\mathbf{A}\left(\mathbf{u}^{n}\right)$. Physically, this means that the material properties lag one time increment behind the solution; recall that $A$ is a function of the dimensionless compressibility and permeability. This interpretation advances the problems to be found, since high variations of material properties occur in the first stages of consolidation. Equation (7) is then reduced to a linear system of algebraic equations

$$
\mathbf{A}\left(\mathbf{u}^{n}\right) \mathbf{u}^{n+1}=\mathbf{u}^{n}+\mathbf{f}
$$

which is easily solved at each time increment.

The main advantage of this technique is that its implementation is extremely simple, but it does, however, present serious drawbacks. The basic hypotheses are that the time increment is sufficiently small and that small increments of time induce negligible modifications in $\mathbf{A}$. Therefore, only under these circumstances can both matrices $\mathbf{A}\left(\mathbf{u}^{n+1}\right)$ and $\mathbf{A}\left(\mathbf{u}^{n}\right)$ be assumed approximately equal. Obviously, this

\section{method may need uneconom \\ over, it should be emphasized \\ Thus, this method require \\ who know from the final resul
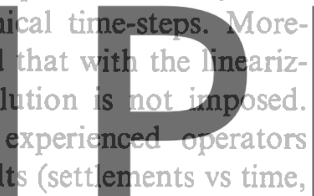

void ratio or pore-water variations with depth, etc.)

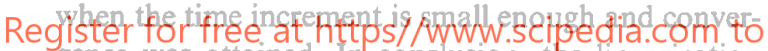
gence was attained. In conclusion, the linearization of the problem is, in spite of its computational simplicity, a dangerous technique for solving eqn (7).

\section{Iterative techniques}

In order to solve the non-linear set of equations, standard non-linear numerical techniques should be implemented. There are a large number of methods for the resolution of eqn (7) which are based on the general scheme defined by

$$
\begin{aligned}
\mathbf{u}_{k+1}^{n+1}=\mathbf{u}_{k}^{n+1}-\left[\mathbf{C}\left(\mathbf{u}_{k}^{n+1}\right)\right]^{-1} & \\
\times & {\left[\mathbf{A}\left(\mathbf{u}_{k}^{n+1}\right) \mathbf{u}_{k}^{n+1}-\left(\mathbf{u}_{k}^{n}+\mathbf{f}\right)\right], }
\end{aligned}
$$

where the subscript $k$ denotes the iteration counter and $\mathbf{C}$ is an arbitrarily chosen matrix. Convergence of the scheme is assumed when the difference between two successive approximations of $\mathbf{u}^{n+1}$ and the residual vector, $\left[\mathbf{A}\left(\mathbf{u}_{k}^{n+1}\right) \mathbf{u}_{k}^{n+1}-\left(\mathbf{u}_{k}^{n}+\mathbf{f}\right)\right]$, are smaller than a predetermined tolerance.

In fact, the choice of $\mathbf{C}$ classifies the numerical technique employed and induces the order of convergence of the method. Therefore, the choice of $\mathbf{C}$ is important and must be done carefully depending on the computational cost (storage and CPU time). Most of the usual techniques take $\mathbf{C}$ as the Jacobian matrix, $\mathbf{J}$, or an approximation to it. The Jacobian matrix for this problem may be written as

$$
\mathbf{J}=\mathbf{A}+\frac{\partial \mathbf{A}}{\partial \mathbf{u}^{n+1}} \mathbf{u}^{n+1}
$$

The first method implemented consists in the following approximation of the Jacobian matrix, $\mathbf{C}=\mathbf{A}$. This method of order one is in fact the well-known 'fixed-point iterative method', which may be written as

$$
\mathbf{A}\left(\mathbf{u}_{k}^{n+1}\right) \mathbf{u}_{k+1}^{n+1}=\mathbf{u}^{n}+\mathbf{f}
$$

and was chosen because its implementation in a linearized computer code is extremely simple. The computer cost per time-step increases because eqn (11) must be constructed and solved, at every instant $t^{n+1}$, for each iteration, $(k+1)$, up to convergence. However, the overall computer cost decreases comparatively with the linearized technique because the time-step may be larger.

In some problems, mostly during filling periods and because of the highly non-linear behavior, convergence is too slow or never obtained. This result may seem negative but it actually gives valuable

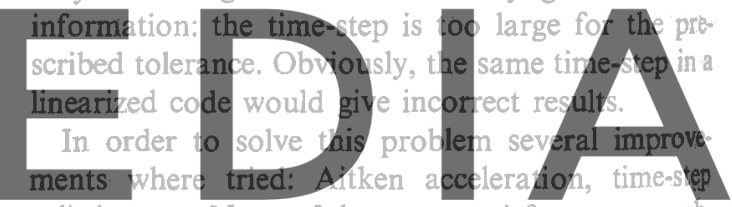

splitting, etc, None of them was satisfactory enough,

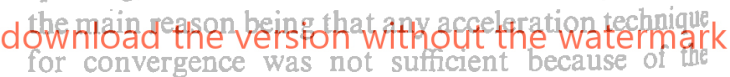
extreme non-linear behavior of the problem.

Consequently, a full Newton-Raphson method is used to avoid the previously cited difficulties for some of the studied problems. This is a second ordet method where the iteration matrix, $\mathbf{C}$, is taken equal to the Jacobian matrix, J. As shown in eqn (10), the derivatives of the coefficients of $A$ with respect to $u_{i}^{n t}$ must be computed to evaluate $\mathrm{J}$. The dependence of these coefficients in $u$ is as follows:

$$
\begin{aligned}
\mathbf{A} & =\mathbf{A}(p, q) \\
p & =p\left(\sigma^{\prime}\right) \\
q & =q(e, k) \\
k & =k(e) \\
e & =e\left(\sigma^{\prime}\right) \\
\sigma^{\prime} & =\sigma^{\prime}(u) .
\end{aligned}
$$

A modification of the computer code is required a modular and structured organization must be usel 
based on the above presented equations. That is, every equation in (12) represents a necessary module where the desired functions and their derivatives are computed. At the end, the coefficients of $\mathbf{A}$ and the Jacobian matrix are obtained. In this manner, modifications of the computer code are relatively simple. For instance, if the numerical scheme changes, only the module associated to eqn (12a) and its derivatives is changed; while, if the constitutive relationships are modified, equs (12d) and (12e) and their derivatives must be updated without perturbation of the general layout of the code. Knowledge of the analytical expressions of eqn (12) and their derivatives renders full Newton-Raphson optimal compared with other high order convergence schemes that do not evaluate J explicitly, such as the quasi-Newton methods.

This second order scheme reaches convergence in a few iterations and for very small tolerances, on the order of $10^{-6}$. Obviously, the computer cost per iteration is larger because of the evaluation of the derivatives. In the linear or first order schemes, the construction of $\mathbf{A}$ takes approximately $70 \%$ of the CPU time while its resolution only uses the $30 \%$ left; here, two matrices are computed in every iteration, thus the cost per iteration almost doubles. However, in general it is less expensive than other techriques because of the important reduction in the number of iterations needed for convergence (three or four iter tions during the first sta roected, the solutions obtaind vith any method are identica. uniqueness of the solution.

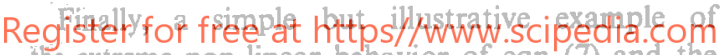
the extreme non-linear behavior of eqn (7) and the importance of adequate initial approximation is shown. Similarly as before, the first time increment of an initial filling analysis over an undrained bed is studied. The top surface is drained and consequently if one node is added per time-step, there is only one unknown: the excess pore-water pressure, $u$, at the bottom. The system of non-linear eqns (7) is now reduced to one equation and the residue can be plotted vs $u$, see Fig. 4. As shown in the figure, only one solution exists for the physical range of $u$. However, it can also be observed that considerable difficulties in the resolution of this problem are induced by a second solution exterior to the domain and the shape of the curve. The developed technique which uses as an initial approximation the solution in the previous step, does the first iteration with the order one scheme and then uses the second order method, converges in a few steps (less than five for tolerances under $10^{-6}$ ) for all the studied cases.

\section{ONE-DIMENSIONAL EXAMPLES}

To show the applicability of the developed computer codes and the advantages of the non-linear

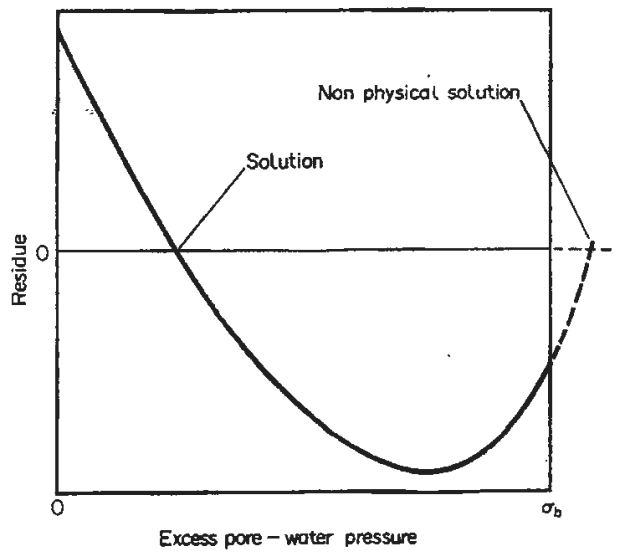

Fig. 4. Residue vs excess pore-water pressure for a one degree of freedom filling analysis.

numerical schemes, two illustrative examples are presented.

The first example is the classical [12] self-weight consolidation of a soil column, starting from uniform conditions (i.e. constant void ratio with depth). This condition is never actually encountered, but it represents laboratory tank tests where filling is "instantaneous" compared with pore pressure dissipation.

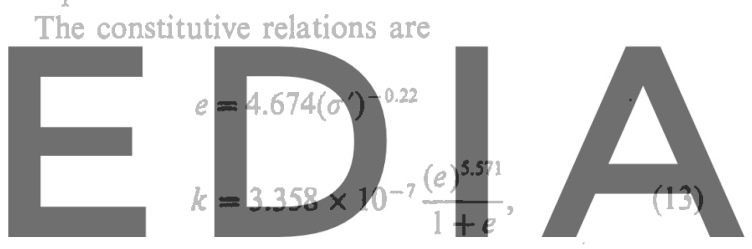

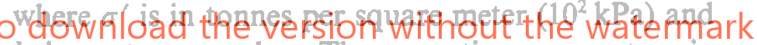
$k$ in meters per day. The operating parameters in eqn (13) are those of one test case considered in [12]. The spatial mesh is composed of 101 nodes and consolidation is simulated over a year.

Figure 5 shows a comparison between consolidation height-time curves. Three analyses are presented: linear model with 20 time-steps (discontinuous line); iterative model with 20 time-steps (continuous line); and iterative model with 1000

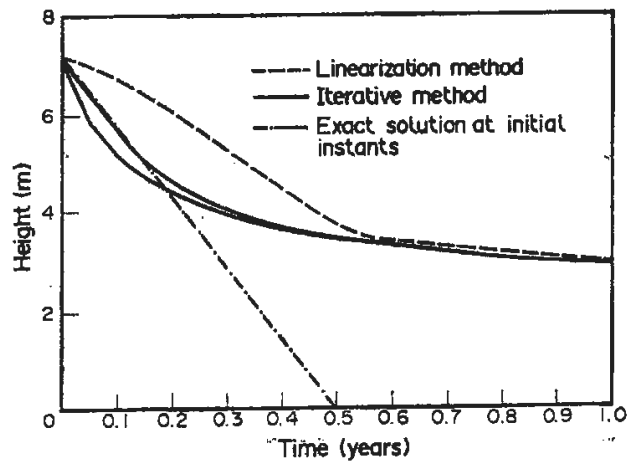

Fig. 5. Height of the deposit vs time. Quiescent consolidation. 


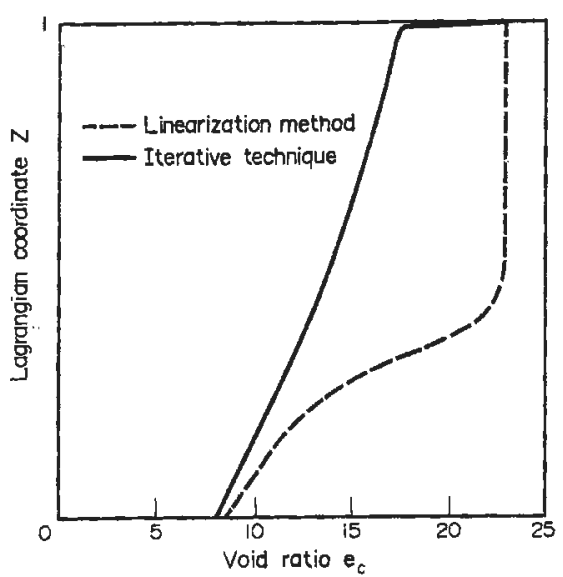

Fig. 6. Comparison between linear and non-linear void ratio distributions after 3 months of quiescent consolidation.

time-steps (continuous line). The linear model clearly underestimates settlement and has an unrealistic change in curvature, while the non-linear model gives similar results to those of a 1000 time-steps analysis. The straight line which represents the theoretical exact initial behavior (constant consolidation rate, see [8]) is in good agreement with both non-linear solutions in spite of the large time increments taken here (20 time-steps per year implies an

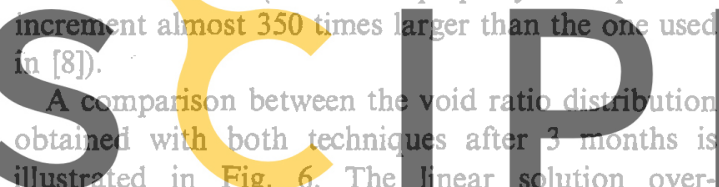
estimates the void ratio in the upper area where the

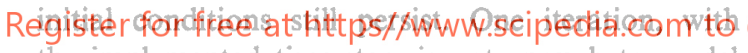
the implemented time-step, is not enough to model the important changes in the void ratio.

It should be noted that, as expected, both methodologies converge to the final conditions which can be evaluated analytically. However, the next example shows that due to the alternation between periods of filling and quiescent consolidation, good accuracy at the first or middle stages of consolidation is needed, because the results obtained after a cycle are the initial conditions for the following one.

By proper manipulation, virtually any sequence of filling and quiescent settling, with or without surcharge, may be simulated. To illustrate the versatility of the computer programs, and their applicability to the prediction of the containment requirements and useful life, a problem composed of four filling and quiescent consolidation phases is shown. The filling periods span over half a year, while the quiescent consolidation takes a year. Three different filling rates have been used: $100 \times 10^{5} \mathrm{~kg} /$ year (initial filling), $300 \times 10^{6} \mathrm{~kg} /$ year (second and third cycles) and $200 \times 10^{6} \mathrm{~kg} /$ year (last cycle); (see Fig. 7à).

Figure 7 presents the height variations with time for different numerical schemes. It compares the linear and non-linear schemes with a time-step of 10 days. It is clearly observed that the linear approach overestimates the needed depth of the deposit, while the non-linear scheme with five nodes per time-step converges clearly to the dash-dotted line computed with a non-linear scheme and time-step of 1 day.

It is obvious that the computer cost per time-step is larger in the non-linear codes. It would therefore seem adequate to compare linear and non-linear results using different time-steps so that both pro. cedures require similar amount of computer time. However, predicting the proper time-step reduction at every instant for the linear analysis is not an easy problem and may be as costly as solving the nonlinear problem itself. This is shown by the fact that the number of iterations varies with time, for a given time increment, in a non-linear simulation.

\section{PSEUDO BI-DIMENSIONAL EXTENSION}

One-dimensional large-strain consolidation is an acceptable model for the overall behavior of a usual waste slurry containment. However, when consolidation is accelerated by vertical drains, or when consolidation occurs in narrow trenches, bi-dimensional effects clearly influence the final solution: Similarly to classical small-strain consolidation, an extension of eqn (7) to bi-dimensional flux and

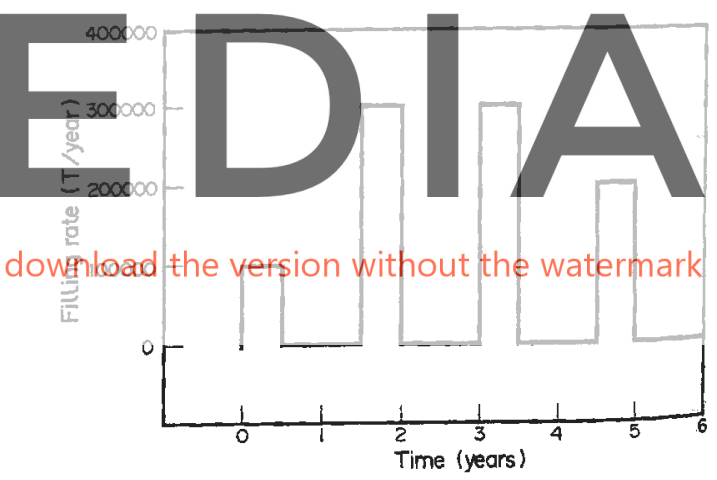

(a)

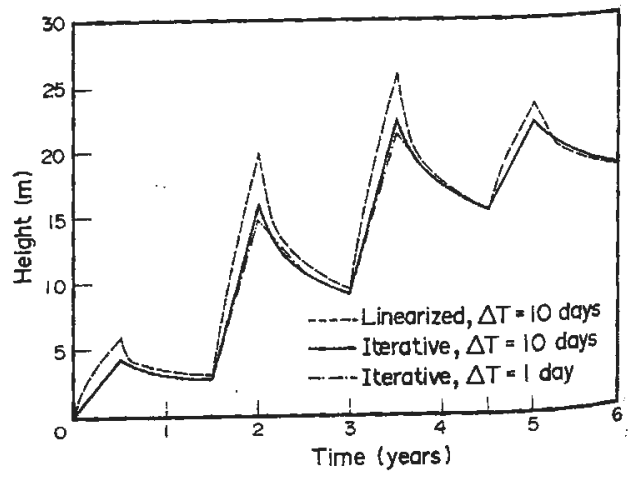

(b)

Fig. 7. (a) Sequence of filling periods and quiescent consali: dation phases. (b) Comparison between different schemes during the simulation of the prediction of the containmet requirements and useful life. 

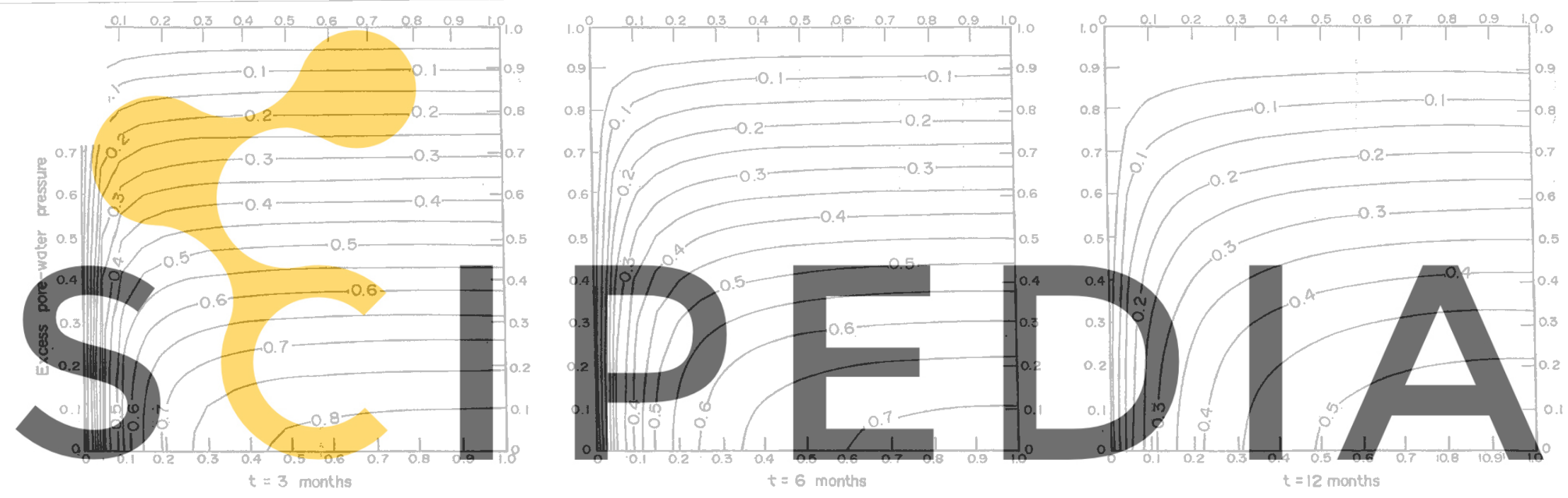

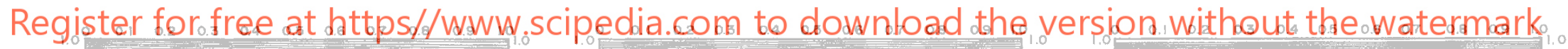
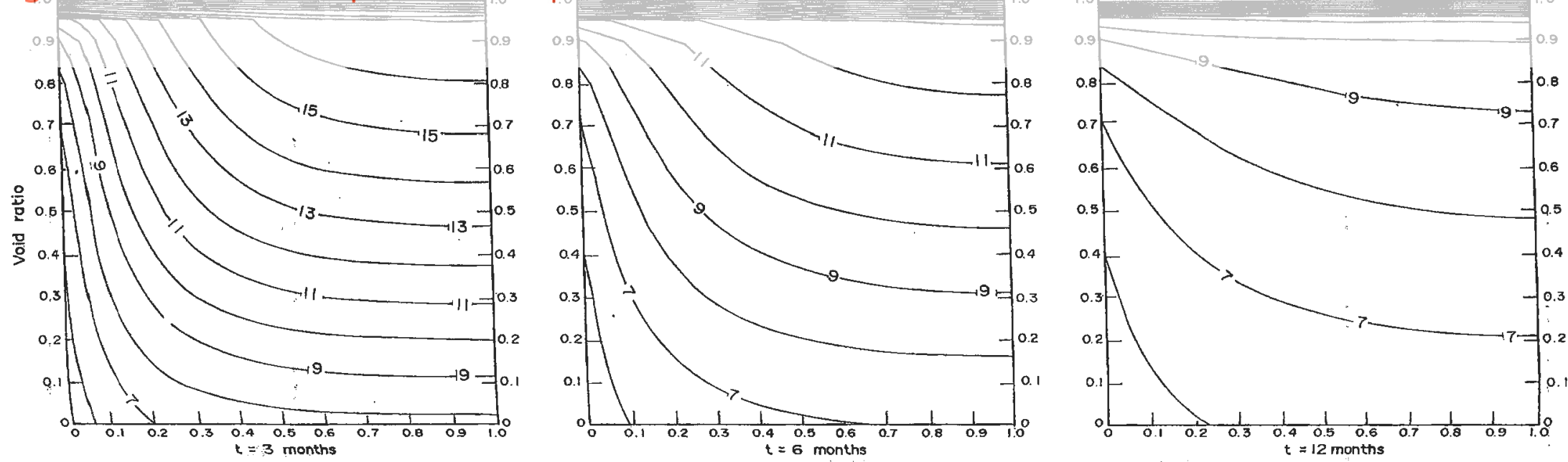

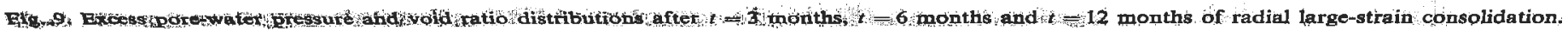


(2) The extremely high variations of the void ratio and coefficient of permeability in the upper area of the deposit must be accurately captured; the proposed multiple node per time-step technique is much more efficient for filling analysis than the classical one node technique.

(3) Since the physical and mathematical aspects of the problem are highly non-linear, non-linear methods rather than the classical linearization method should be employed; the latter does not assure convergence, may imply uneconomical timesteps and, in general, overestimates the void ratio thus underestimating settlement and strength (this is important for reclamation purposes).

(4) A second order non-linear method (NewtonRaphson) is necessary to ascertain convergence, this provides a robust and efficient algorithm; however, a good initial approximation of the solution and a first iteration with the 'fixed-point iterative method' are also recommended.

(5) The bi-dimensional extension amplifies the non-linear aspects, however the 'split-step method' allows a ready generalization of the one-dimensional techniques, and successful results are also obtained with the developed non-linear algorithm.

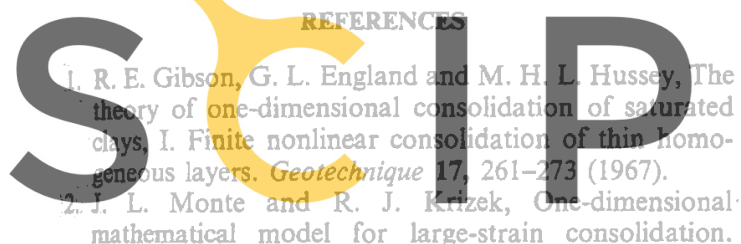

3. F. Somogyi, Analysis and prediction of phosphatic clay consolidation: implementation package. Technical report; Florida Phosphatic Clay Research Project, Lakeland, FL (1979).

4. R. L. Schiffman, Finite and infinitesimal strain consolidation. J. Geotech. Engng Div., ASCE 106, 203-207 (1980).

5. R. E. Gibson, R. L. Schiffman and K. W. Cargill, The theory of one-dimensional consolidation of saturated clays, II. Finite nonlinear consolidation of thick homogeneous layers. Can. Geotech. J. 18, 280-293 (1981).

6. R. J. Krizek and F. Somogyi, Perspectives on modelling consolidation of dredged materials. Proceedings of the Symposium on Sedimentation/Consolidation Models, ASCE, San Francisco, CA, pp. 296-332 (1984).

7. R. L. Schiffman, V. Pane and V. Sunara, Sedimentation and consolidation. Proceedings of the Symposium on Sedimentation/Consolidation Models, ASCE, San Francisco, CA, pp. 57-121 (1984).

8. J. R. Feldkamp, Numerical analysis of one-dimensional nonlinear large-strain consolidation by the finite element method. J. Porous Media 4, 239-257 (1989).

9. S. D. Koppula and Morgenstern, On the consolidation of sedimenting clays. Can. Geotech. J. 19, 260-268 (1982).

10. W. D. Carrier, L. G. Bromwell and F. Somogyi, Design capacity of slurried mineral waste ponds. J. Georech. Engng Div., ASCE 109, 699-716 (1983).

11. A. Huerta, G. A. Kriegsmann and R. J. Krizek, Permeability and compressibility of slurries from seepageinduced consolidation. J. Geotech. Engng, ASCE I14, 614-627 (1988)

12. Symposium on Consolidation and Disposal of Phosphatic and Other Waste Clays. Department of

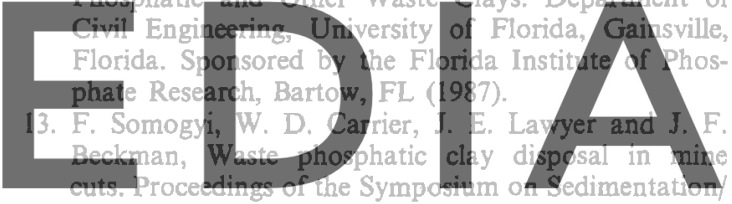

Consolidation Models, ASCE, San Francisco, CA Cos lidation Models, ASCE, San Francisco, CA 